\title{
Transcatheter aortic valve implantation: status update
}

\author{
Antoinette Neylon ${ }^{1}$, Khalid Ahmed ${ }^{1}$, Federico Mercanti ${ }^{1}$, Faisal Sharif ${ }^{1,2}$, Darren Mylotte ${ }^{1,2}$ \\ ${ }^{1}$ Department of Cardiology, University Hospital of Galway, Newcastle Road, Galway, Ireland; ${ }^{2}$ National University of Ireland, Galway, Ireland \\ Contributions: (I) Conception and design: All authors; (II) Administrative support: None; (III) Provision of study materials or patients: None; (IV) \\ Collection and assembly of data: None; (V) Data analysis and interpretation: None; (VI) Manuscript writing: All authors; (VII) Final approval of \\ manuscript: All authors. \\ Correspondence to: Darren Mylotte, BCh MB, MD. Department of Cardiology, University Hospital of Galway, Newcastle Road, Galway, Ireland. \\ Email: darrenmylotte@gmail.com.
}

\begin{abstract}
Transcatheter aortic valve implantation (TAVI) has emerged as the gold standard technique for all patients with symptomatic severe aortic stenosis at elevated surgical risk. Much progress has been made to reduce procedural complications and improve patient outcomes. The impressive results of contemporary TAVI can be attributed to a variety of factors, including improving operator experience, pre-operative patient screening, and developments in transcatheter heart valve and delivery system technology. Despite these advances, serious procedural complications continue to occur and there remain some anatomical subsets and patient groups to whom TAVI technology has not been expanded. Herein we discuss these unmet needs in TAVI.
\end{abstract}

Keywords: Transcatheter aortic valve implantation (TAVI); aortic stenosis; surgical aortic valve replacement (SAVR)

Submitted Sep 27, 2018. Accepted for publication Oct 11, 2018.

doi: $10.21037 /$ jtd.2018.10.34

View this article at: http://dx.doi.org/10.21037/jtd.2018.10.34

\section{Introduction}

Transcatheter aortic valve implantation (TAVI) has emerged as a gold standard therapeutic option for patients with symptomatic severe aortic stenosis who are ineligible for conventional surgical aortic valve replacement (SAVR) or at high- or intermediate-operative risk (1). The last decade has witnessed a succession of randomized clinical trials comparing TAVI to SAVR which, in addition to reporting equivalent mortality and stroke rate between these therapies, have also documented impressive reductions in important complications associated with TAVI $(2,3)$. This progress can be attributed to improving operator experience, innovation of transcatheter heart valves and delivery catheters (Figure 1), the expanded use of 3-dimensional (3D) imaging including multislice computed tomography, and the extension of TAVI technology to younger and lower risk patients. In order to compete with or surpass the excellent results of SAVR in younger patients, further refinements of transcatheter aortic valves are required. Moreover, there remain several patient subsets in whom TAVI remains challenging and extending this technology to these patients will require iterative change. Herein, we discuss potential patient subsets to which TAVI can be extended and discuss what device changes are required to meet the emerging needs.

\section{Low surgical risk}

To date, six randomized trials have compared TAVI to medical therapy in inoperable patients (4), and TAVI to SAVR in patients at high $(5,6)$ intermediate $(2,3)$ and low surgical risk (7). When considered together, these studies demonstrate that the rates of death and/or stroke are reduced/similar in TAVI compared to the historical gold standard. Moreover, TAVI is associated with lower rates of bleeding, acute kidney injury, atrial fibrillation (8). On the other hand, SAVR is associated with lower rates of paravalvular leak and permanent pacemaker (PPM) than TAVI (8). Based on this information, European guidelines 

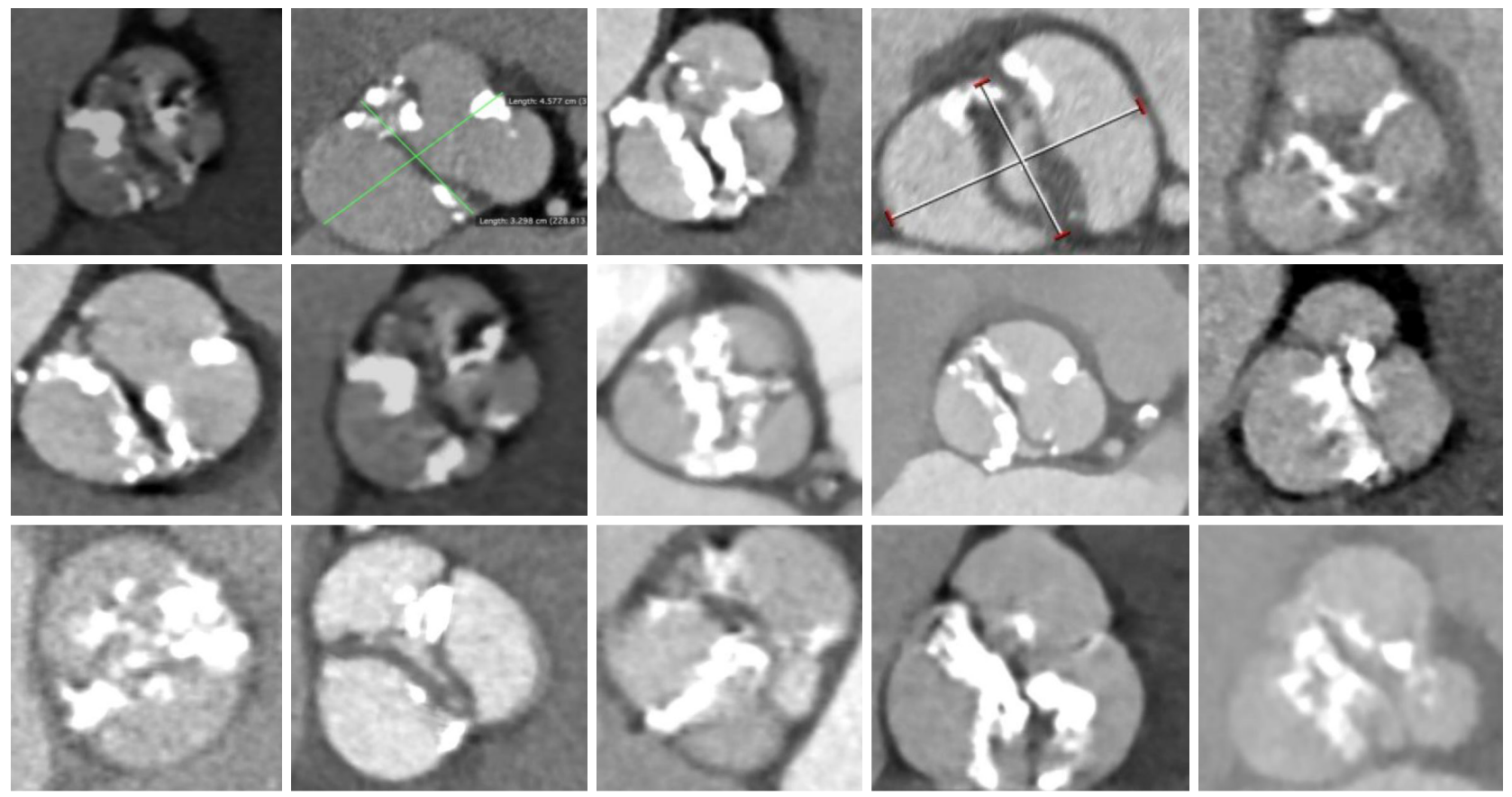

Figure 1 Bicuspid aortic valve. The tremendous variability of bicuspid aortic valve disease imaged with multislice computed tomography.

recommend TAVI as a Class $1 \mathrm{~A}$ indication for the treatment of symptomatic severe aortic stenosis in patients at elevated operative risk (1).

Extending TAVI to patients at lower operative risk is currently controversial and is the subject of three ongoing randomized controlled trials (PARTNER 3 (NCT02675114), CoreValve Low Risk (NCT02701283), and NOTION 2 (NCT02825134). One important issue to consider when discussing risk categorization of TAVI patients, is that to date, all studies have assigned procedural risk using surgical risk scores with poor discrimination for adverse events in TAVI populations (9). Recognising this issue, the European Valvular Heart Disease guidelines recommend a Heart Team discussion of individualized patient risk rather than using risk scores to determine treatment allocation in aortic stenosis (1). Importantly, low risk does not necessarily imply younger age: the average age in all comparative randomized trials to date has been $>80$ years old and even the low-risk NOTION trial recruited patients with a mean age of 81.8 years. The totality of available evidence confirms that TAVI works very well in elderly patients (irrespective of risk categorization). One could therefore speculate that little will be learned from the ongoing low-risk studies if they recruit patients of 80 years of age. Among the three aforementioned low-risk trials, only the NOTION 2 study has an upper age limit (75 years).

Younger age is a key issue when considering extension of TAVI to "lower-risk" patients as the surgical literature is replete with studies reporting that younger age is closely associated with early structural valve failure (SVF) (10). A standardized definition of SVF has only recently been published and will help the community compare surgical and percutaneous strategies henceforth (11). Among younger patients the durability of transcatheter heart valve technology remains largely untested and thus extending TAVI to younger patients will remain contentious until longterm durability data are available. Although some longer-term (8 years) data has emerged and reports acceptable rates of SVF for transcatheter valve technology (12), this information does not apply to younger patients where accelerated SVF can be expected. The field will therefore have to wait until the longer term results (10 years) of the NOTION 2 trail are reported to fully understand the implications of extending TAVI to younger patients.

\section{Conduction abnormalities}

Conduction system abnormalities continue to be a relatively common complication following TAVI. The mechanism of injury of the conduction system during TAVI relates 
to the proximity of the left ventricular outflow tract (implantation site for TAVI) and the AV-node/left bundle branch. Several factors have been associated with the requirement for the implantation of a new PPM after TAVI, including: pre-existing conduction system disease, volume of left ventricular outflow tract calcification, type of THV, depth of deployment, degree of THV oversizing relative to the native anatomy, and the length of the membranous interventricular septum (13).

The rate of new PPM varies according to the factors described above and ranges from $8.5 \%$ with balloonexpandable Edwards SAPIEN 3 (Edwards Lifesciences, Irvine, CA, USA) valve in the PARTNER 2 study to $35.5 \%$ with the mechanical-expandable Boston Scientific Lotus valve $(2,14)$. The clinical implications of new PPM post TAVR remain a matter of conjecture. Most believe however that there may be important repercussions associated with right ventricular pacing: long-term reduction in left ventricular ejection fraction and cardiac output (15). These adverse effects could have particular implications for younger patients that would be exposed to decades of right ventricular pacing post TAVI. Certainly, new PPM implantation after TAVI increases the length of hospitalization, the cost of the procedure, and exposes patients to the risk of another intervention.

Efforts to reduce the incidence of new PPM have been successful: the target depth for implantation of the SAPIEN 3, Lotus (Boston Scientific Corp, Marlborough, Massachusetts USA), and CoreValve Evolut R/PRO (Medtronic, Minneapolis, Minnesota, USA) valves has been reduced from $5-8$ to $3-5 \mathrm{~mm}$; the rate of balloonpredilatation has fallen; and new prostheses (ACRUATE NEO, Boston Scientific Corp., Marlborough, Massachusetts USA) associated with very low rates of PPM have emerged (16). Novel mechanisms of anchoring the THV, such as leaflet clipping used by the JenaValve (JenaValve Technology, Irvine, CA, USA), have the potential to further reduce the rates of conduction disturbance post TAVI (17).

\section{Bicuspid aortic valve (BAV)}

$\mathrm{BAVs}$ are a frequently encountered congenital abnormality, occurring in approximately $1 \%$ of the general population (18). $\mathrm{BAV}$ stenosis and/or regurgitation is the most common indication for SAVR in patients $<70$ years of age but bicuspid morphology has served as an exclusion criterion for all randomized trials of TAVI. The exclusion of bicuspid anatomy relates to several anatomic features which render these valves more difficult to treat percutaneously: leaflet calcification is more extensive, extending from the base to the tips of the leaflets; there may be a raphe present which is often heavily calcified; commissural fusion is common; the sinuses of Valsalva are asymmetrical; aortopathy and associated aortic incompetence occurs frequently; the annuli are often larger than tricuspid valves (Figure 2). Bicuspid morphology is also more commonly encountered in younger patients and ultimately the extension of TAVI to the young will probably be determined by acute and longterm results in bicuspid morphology.

Early reports of TAVI in bicuspid morphology described high incidences of paravalvular leak and the requirement for a second THV due to malposition (19). The advent of repositionable TAVI devices and multislice CT has however resulted in improvements in procedural and clinical outcomes. Yoon et al. have recently demonstrated equivalent outcomes for contemporary TAVI between bicuspid and tricuspid aortic valve stenosis (20). There remain however important questions regarding TAVI in bicuspid morphology: (I) how should we size the THV? The extensive calcification and frequent presence of a raphe have led to calls for an alternate sizing algorithm in bicuspid valves. The most accurate and reproducible method of sizing the "supra-annular" portions of the aortic root have not been elucidated, but studies such as the prospective BIVOLUT $\mathrm{X}$ registry will advance the field. (II) How durable are THVs after implantation in bicuspid morphology? (III) Is balloon pre-dilatation mandatory? (IV) Is there a higher incidence of conduction disturbance in bicuspid anatomy? (V) What is the optimal THV type in this anatomy (balloon-, self-, or mechanical-expanding)?

Foremost among these concerns is the issue of THV durability in bicuspid anatomy. The current trend of supraannular sizing the THV often (not always) results in a smaller valve size being selected compared to annular sizing. Since the haemodynamics of a smaller valve would be expected to be less favourable than those a larger valve, it is possible that TAVI in bicuspid anatomy could lose the haemodynamic advantage that it enjoys over SAVR in tricuspid valves. If accelerated SVF is observed with TAVI in bicuspid cohorts, then SAVR would continue as the dominant treatment strategy. Dedicated trials of TAVI compared to SAVR in bicuspid morphology are required. 
A

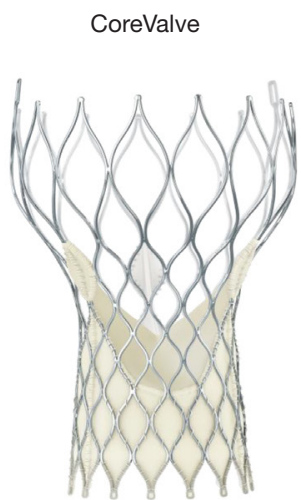

B

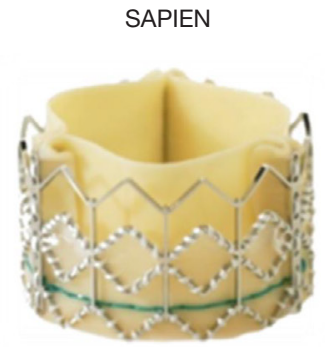

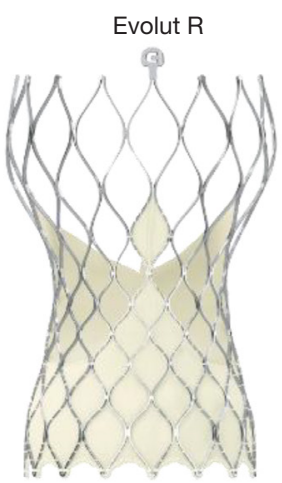
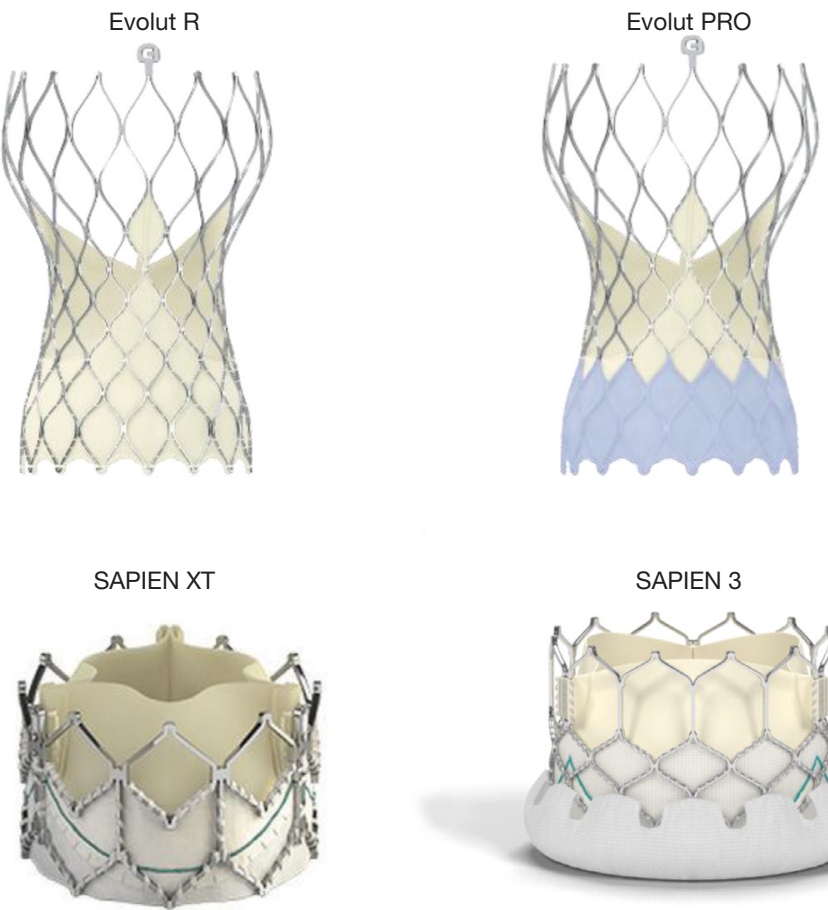

SAPIEN 3

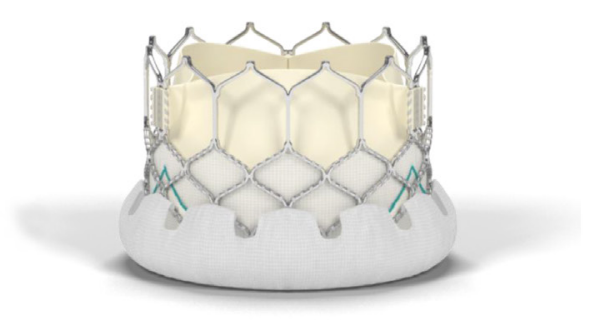

Figure 2 Transcatheter aortic valve development. Iterations of the (A) Medtronic CoreValve and (B) Edwards SAPIEN transcatheter heart valves.

\section{Pure aortic regurgitation}

In the Euro Heart Survey on Valvular Heart Disease, only one-fifth of patients with severe native aortic incompetence and reduced left ventricular ejection fraction were referred for SAVR (21). Thus, there is an important clinical need for transcatheter therapies for these patients. Unlike aortic stenosis however, aortic incompetence can encompass a variety of distinct pathologies of the aortic valve, root, or the left ventricle. Severely regurgitant valves often arise from infective endocarditis, bicuspid morphology, or collagen disorders, and are associated with increased left ventricular dimensions and reduced ejection fraction. Moreover, the predominant forces on the THV in aortic stenosis are from the left ventricle into the aorta, but are reversed in aortic regurgitation. This fundamental difference poses an important challenge for TAVI.

The proportion of THVs used to treat purely regurgitant aortic valves remains low $(<1.5 \%$ of all TAVI procedures) (22). These cases remain difficult: malposition requiring a second TVH; > moderate PVL; longer procedures with more radiation exposure and contrast used; and ultimately VARC-defined device success in only $72 \%$ (22). Contemporary THVs are more successful than first generation devices but dedicated valves systems will likely be required to master the native pure regurgitant field. The JenaValve is the only THV that has been approved in Europe for the treatment of aortic regurgitation. This device has a leaflet clipping mechanism for anchoring and does therefore not rely on radial force to anchor and seal. Early results with the transapical version of this deice were promising and further data with the new transfemoral delivery system are eagerly awaited $(17,23)$.

\section{Coronary artery occlusion}

Coronary artery occlusion is a devastating complication of TAVI (Figure 3). Although relatively uncommon in contemporary TAVI practice $(<1 \%)$, coronary occlusion carries a 30 -day mortality of up to $40 \%$ (24). Ostial coronary artery occlusion occurs due to displacement of bulky native leaflet tissue towards the coronary ostium with direct obstruction of coronary flow or due to contact of the displaced leaflet tissue with the sinotubular junction and indirectly reduction of coronary flow by sealing off the 

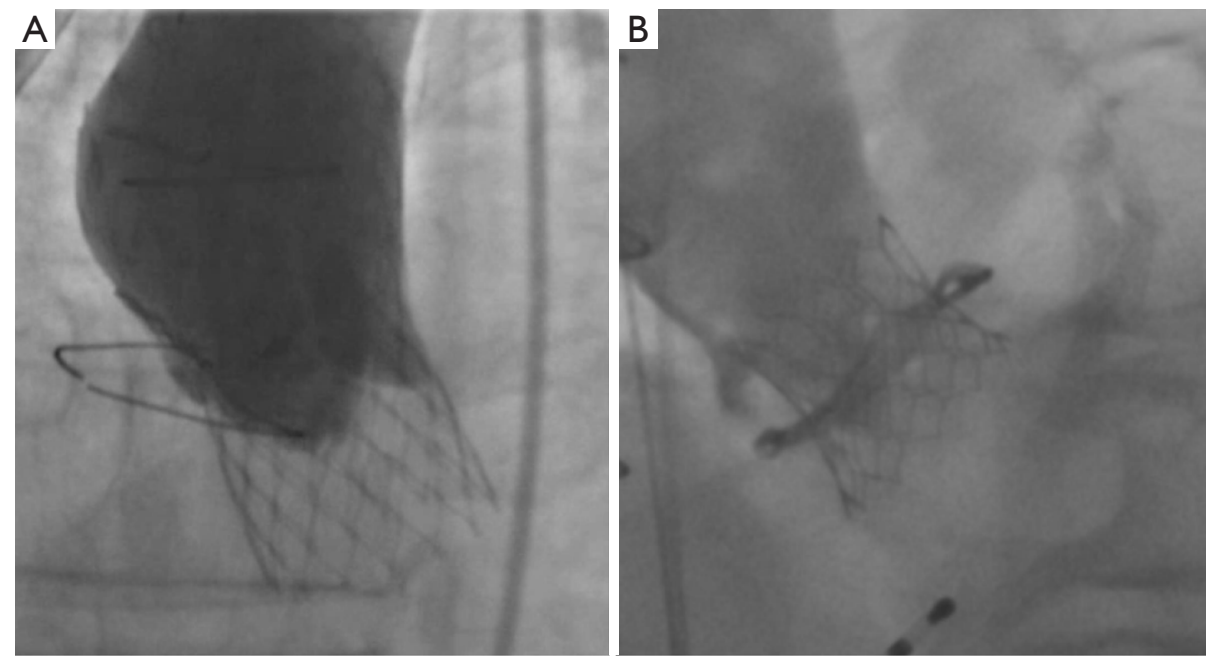

Figure 3 Coronary occlusion after TAVI. Coronary occlusion after TAVI is a devastating complication. TAVI, transcatheter aortic valve implantation.

coronary sinuses.

Low-lying coronary ostia $(<12 \mathrm{~mm})$, shallow sinuses of Valsalva $(<30 \mathrm{~mm})$, the use of balloon-expandable THVs, and transcatheter aortic valve within failed surgical aortic valve (TAV in SAV) procedures are predictors of coronary obstruction (24). The incidence of coronary occlusion during TAV-in SAV procedures is particularly high with stentless bioprostheses or with stented surgical valves with externally mounted leaflets $(2.3-3.5 \%)$, and is associated with 30 -day mortality rates of up to $50 \%$ $(24,25)$.

In cases deemed at high risk for coronary obstruction, coronary "protection" is usually undertaken by positioning an undeployed coronary stent in the at-risk artery prior to THV deployment $(26,27)$. If coronary blood flow is reduced after THV deployment, the stent is retracted to extend from the coronary ostia parallel to the THV and is subsequently deployed to create a channel between the displaced leaflets and the aortic root. This technique is known as a "chimney" stenting and although it is potentially life-saving, there is no information available on the long-term outcomes of these cases.

Bioprosthetic or native aortic scallop intentional laceration to prevent coronary artery obstruction (BASILICA) is a novel technique which aims to intentionally lacerate a valve leaflet to create a triangular space for coronary or sinus of Valsalva blood flow after THV implantation (28). A U.S. safety and feasibility study of this novel technique is underway.

\section{Maintaining coronary access after TAVI}

Most THVs have the potential to impede access to the native coronary arteries once implanted. Of course, valves with a lower frame height (SAPIEN 3, Lotus) are less likely to impair access than those with tall frames which extend beyond the sinotubular junction (CoreValve, Portico, NVT, Acurate). If a THV frame does extend above the coronary arteries, then coronary access can be determined by the width of the sinuses of Valsalva, the size of the THV cells through which a coronary catheter can be manipulated, the height of the internal/external THV skirts, and by the position of the commissural posts of the THV. Manufactures of THVs have made efforts to reduce the density of metal in the upper regions of their THV frames to facilitate coronary access but selective engagement of the coronary arteries can be difficult and has been reported to impede emergent life-saving percutaneous coronary interventions $(29,30)$. When performing SAVR, surgeons will orientate the surgical bioprosthesis to the same position as the native valve commissures. In contrast, there is currently no way to orientate a THV with the native commissures and thus valve posts can be directly placed in front of the coronary arteries and hamper coronary access, even when the frame cells can be negotiated. When TAVI is extended to younger patients and TAV-in-TAV procedures are required, access to the coronary arteries may become impossible as the degenerated leaflets of the initial THV will be pinned to the frame, creating a stent graft at the level of 
the aortic root. The prevalence of this particular issue and the development of novel solutions may have a large influence on the extension of TAVI technology to younger patients.

\section{Vascular access}

In the original PARTNER trials of balloon-expandable TAVI, half of all cases were performed using transapical vascular access (5). Thoracic access has more recently been associated with increased ICU and hospital stay, more bleeding and requirement for transfusion, and increased mortality. The landmark meta-analysis of randomized TAVI trials by Siontis et al. clearly documents that the advantage of TAVI compared to SAVR is driven by transfemoral (versus thoracic) vascular access (8). Gratifyingly, more than $90 \%$ of all TAVI cases are currently performed via the transfemoral route. If femoral vascular access is not possible, then subclavian, carotid, and transcaval routes are usually favoured in order to avoid the protracted recovery associated with thoracic access.

The widespread adoption of multislice CT to screen potential vascular routes and increasing operator experience managing large bore vascular access has seen the incidence of vascular complications fall. Industry has responded to the need to perform more procedures via the femoral route, and the routine use of 22-24 Fr vascular access sheaths has been replaced by a variety of smaller diameter solutions: Medtronic In-Line sheath (14-16 Fr); Edwards Lifesciences expandable sheath (14-16 Fr); Boston Scientific I-Sleeve (18 Fr); Terumo Solopath (14-21 Fr).

The incidence of major vascular complications in contemporary TAVI practice is now $<5 \%$ (31). Delivery system development continues to focus on reduction in sheath size, but there is always a trade-off with respect to additional features: for example, the Medtronic Evolut PRO valve has increased the size of the delivery system from 14 to $16 \mathrm{Fr}$ to accommodate an adaptive seal which reduces paravalvular leak. Innovative approaches such as the development transcaval and percutaneous transaxillary vascular access routes will however provide fully percutaneous options for patients that cannot undergo transfemoral TAVI $(32,33)$. More work is required however to increase the proportion of patients that can benefit from transfemoral TAVI and to further reduce the risk of vascular complications.

\section{Cost}

Transcatheter heart valves are expensive. The enormous variability of the application of this innovative technology across the globe can largely be attributed to divergent healthcare spending among nations (34). It is interesting to speculate that as new companies enter the market that the price of devices will fall; however in many parts of the globe, the cost of THVs remains largely unchanged since commercialization in 2007. Important changes in the execution of TAVI have made the procedure more cost effective. In fact, a recent cost-effectiveness analysis of the PARTNER IIA study has demonstrated that TAVI is more cost effective than surgery (35). This is especially true for transfemoral procedures relative to transthoracic access, which was more costly than SAVR. Interestingly, it was the reduced post hospital discharge costs that made TAVI a dominant strategy compared to SAVR in this analysis. The PARTNER IIA trial was performed in the U.S. where most TAVI continues to be performed with a full heart team under general anaesthesia. In Europe, TAVI is now routinely performed under local anaesthesia, without the requirement for transesophageal echocardiography, without a surgeon and/or a theatre team present. The average duration of ICU and hospital stay has consequently been reduced and the cost of the procedure has fallen (36). Simplified TAVI will further reduce procedural costs, but as TAVI extends into the large population of patients at intermediate and low surgical risk, the requirement to reduce the costs of the $\mathrm{THV}$ itself is ever more acute; recent estimates the suggest that the number of potential TAVI candidates to be 114,757 (95\% CI: 69,380-172,799) in Europe and 58,556 (95\% CI: $35,631-87,738)$ in Northern-America per annum (37).

\section{Anticoagulation after TAVI}

The optimal anticoagulant and antiplatelet strategy after TAVI is unknown. The aim of anticoagulants in the setting of TAVI is to reduce the risk of cerebrovascular events, which could arise in the periprocedural or postprocedural periods. In the post-procedural period, stroke could be attributed to pre-existing or new atrial fibrillation or the development of thrombus on the frame of the THV or on the valve leaflets. The latter scenario is of particular relevance with the recent identification of subclinical leaflet thrombosis in a significant proportion of patients undergoing TAVI (average 13\%; range $8-30 \%$ of contemporary THVs) (38). The impact of subclinical leaflet thrombosis remains unknown and to date, this phenomenon has not been associated with an increase in cerebrovascular events (38). Importantly, leaflet thrombosis 
does not occur in patients treated with oral anticoagulants. In contrast, dual antiplatelet therapy (DAPT) does not prevent leaflet thrombosis. Current guidelines recommend the use DAPT (aspirin and clopidogrel) for a period of 3-6 months post TAVI in patients without an indication for oral anticoagulants (38). This practice is not supported by any meaningful data and is based on recommendations for DAPT after percutaneous coronary intervention and has been questioned since DAPT does not prevent leaflet thrombosis but is clearly associated with an increased bleeding risk in the elderly and frail TAVI population. Nonaccess site bleeding (mostly intracranial and gastrointestinal) occurs in up to $20 \%$ of TAVI patients at 5 years and is associated with increased mortality (39). Moreover, the only study comparing single and DAPT after TAVI suggested net clinical harm with the DAPT strategy (40). Furthermore, a patient-level meta-analysis of 421 patients from 3 openlabel randomized controlled trial of DAPT versus SAPT reported a significant increase in life-threatening bleeding with DAPT (6.7\% vs. 2.4\%, $\mathrm{P}=0.036)$ (41).

Consistent with the uncertainty in this area, a recent survey of 250 TAVI centres in 38 countries showed wide variability in the antiplatelet treatment regimens used after TAVI: however, the most commonly used strategy was DAPT for 3-6 months (74\%) (42). Several ongoing clinical trials will help to establish the optimal antiplatelet/ anticoagulant strategy after TAVI. These studies are comparing single and DAPT with oral anticoagulation using Coumadin or the novel oral anticoagulants. The GALILEO trial is comparing a strategy of 1 month DAPT and then single antiplatelet therapy for 18 months to a regimen of 1 month rivaroxaban $10 \mathrm{mg}$ and aspirin and then Rivaroxaban $10 \mathrm{mg}$ only for 18 months, has been stopped early by the data safety management board due to an excess of major bleeding in NOAC-treated patients. Thus, there remains considerable uncertainty among practicing clinicians regarding the balance between preventing ischaemic/embolic and bleeding events after TAVI.

\section{Cerebral embolic protection}

Stroke prevention is perhaps the most important issue for patients considering TAVI. Stroke rates in randomized trials, where systematic neurological assessment before and after TAVI is mandated, range from $3.4 \%$ to $4.8 \%$ $(3,14)$. These rates exceed those reported in registry data simply because small neurological deficits are less likely to be noticed in day-to-day clinical practice. Half of all stroke events occur within 72 hours of the index TAVI procedure and specific technical refinements therefore have the potential to reduce procedural stroke (42). A variety of cerebral embolic protection devices have been developed but only one, the Sentinel cerebral protection system (Boston Scientific Corp, Marlborough, Massachusetts USA) has been approved by the US FDA. The available data on this device divides opinion in the TAVI community: there are "believers" and "non-believers". These divergent opinions stem from the absence of clinical trial data demonstrating a meaningful reduction in stroke prevention with this technology. The Sentinel IDE study randomized 240 patients to cerebral protection or standard therapy and the primary efficacy endpoint was new lesion volume on serial cerebral magnetic resonance imaging (43). The device was proven to be safe but did not significantly reduce new lesion volume in this study. A patient level meta-analysis of 5 randomized trials of cerebral embolic protection, including 623 patients, has also failed to report a significant reduction in clinical stroke events (44). The jury remains out therefore on the efficacy of cerebral embolic protection in TAVI and this technology will probably remain controversial until tested in a large randomized trial powered to detect a reduction in clinical stroke.

\section{Conclusions}

TAVI has developed at a tremendous pace over the last decade and has become a gold standard therapy for patients with symptomatic severe aortic stenosis. The extension of this technology to younger patients and those with more challenging anatomy must be based on evidence of equivalent safety and efficacy to SAVR. Novel devices and procedural strategies will be required to overcome existing issues, such as conduction disturbance and coronary access. Further study is necessary to define the optimal stroke prevention strategy. Reducing cost is paramount in the current cost-constrained environment. There remains much work to do.

\section{Acknowledgements}

None.

\section{Footnote}

Conflicts of Interest: D Mylotte is Consultant and Proctor for Medtronic and Microport. The other authors have no conflicts of interest to declare. 


\section{References}

1. Baumgartner H, Falk V, Bax JJ, et al. 2017 ESC/EACTS Guidelines for the management of valvular heart disease. Eur Heart J 2017;38:2739-91.

2. Leon MB, Smith CR, Mack MJ, et al. Transcatheter or Surgical Aortic-Valve Replacement in Intermediate-Risk Patients. New Engl J Med 2016;374:1609-20.

3. Reardon MJ, Van Mieghem NM, Popma JJ, et al. Surgical or Transcatheter Aortic-Valve Replacement in Intermediate-Risk Patients. New Engl J Med 2017;376:1321-31.

4. Leon MB, Smith CR, Mack M, et al. Transcatheter aortic-valve implantation for aortic stenosis in patients who cannot undergo surgery. New Engl J Med 2010;363:1597-607.

5. Smith CR, Leon MB, Mack MJ, et al. Transcatheter versus surgical aortic-valve replacement in high-risk patients. New Engl J Med 2011;364:2187-98.

6. Adams DH, Popma JJ, Reardon MJ, et al. Transcatheter aortic-valve replacement with a self-expanding prosthesis. New Engl J Med 2014;370:1790-8.

7. Thyregod HG, Steinbruchel DA, Ihlemann N, et al. Transcatheter Versus Surgical Aortic Valve Replacement in Patients With Severe Aortic Valve Stenosis: 1-Year Results From the All-Comers NOTION Randomized Clinical Trial. J Am Coll Cardiol 2015;65:2184-94.

8. Siontis GC, Praz F, Pilgrim T, et al. Transcatheter aortic valve implantation vs. surgical aortic valve replacement for treatment of severe aortic stenosis: a meta-analysis of randomized trials. Eur Heart J 2016;37:3503-12.

9. Mylotte D, Piazza N. Patient selection for transcatheter or surgical intervention: the Heart Team TRUMPS the STS. EuroIntervention 2016;12:1439-40.

10. Mylotte D, Osnabrugge RL, Martucci G, et al. Failing surgical bioprosthesis in aortic and mitral position. EuroIntervention 2013;9 Suppl:S77-83.

11. Capodanno D, Petronio AS, Prendergast B, et al. Standardized definitions of structural deterioration and valve failure in assessing long-term durability of transcatheter and surgical aortic bioprosthetic valves: a consensus statement from the European Association of Percutaneous Cardiovascular Interventions (EAPCI) endorsed by the European Society of Cardiology (ESC) and the European Association for Cardio-Thoracic Surgery (EACTS). Eur Heart J 2017;38:3382-90.

12. Eltchaninoff H, Durand E, Avinee G, et al. Assessment of structural valve deterioration of transcatheter aortic bioprosthetic balloon-expandable valves using the new European consensus definition. EuroIntervention 2018;14:e264-e271.

13. Bob-Manuel T, Nanda A, Latham S, et al. Permanent pacemaker insertion in patients with conduction abnormalities post transcatheter aortic valve replacement: a review and proposed guidelines. Ann Transl Med 2018;6:11.

14. Feldman TE, Reardon MJ, Rajagopal V, et al. Effect of Mechanically Expanded vs Self-Expanding Transcatheter Aortic Valve Replacement on Mortality and Major Adverse Clinical Events in High-Risk Patients With Aortic Stenosis: The REPRISE III Randomized Clinical Trial. JAMA 2018;319:27-37.

15. Wilkoff BL, Cook JR, Epstein AE, et al. Dual-chamber pacing or ventricular backup pacing in patients with an implantable defibrillator: the Dual Chamber and VVI Implantable Defibrillator (DAVID) Trial. JAMA 2002;288:3115-23.

16. Möllmann H, Hengstenberg C, Hilker M, et al. Realworld experience using the ACURATE neo prosthesis: 30 day outcomes of 1,000 patients enrolled in the SAVI TF registry. EuroIntervention 2018;13:e1764-70.

17. Schäfer U, Schirmer J, Niklas S, et al. First-in-human implantation of a novel transfemoral selfexpanding transcatheter heart valve to treat pure aortic regurgitation. EuroIntervention 2017;13:1296-9.

18. Siu SC, Silversides CK. Bicuspid aortic valve disease. J Am Coll Cardiol 2010;5 5:2789-800.

19. Mylotte D, Lefevre T, Sondergaard L, et al. Transcatheter aortic valve replacement in bicuspid aortic valve disease. J Am Coll Cardiol 2014;64:2330-9.

20. Yoon SH, Bleiziffer S, De Backer O, et al. Outcomes in Transcatheter Aortic Valve Replacement for Bicuspid Versus Tricuspid Aortic Valve Stenosis. J Am Coll Cardiol 2017;69:2579-89.

21. Iung B, Baron G, Butchart EG, et al. A prospective survey of patients with valvular heart disease in Europe: The Euro Heart Survey on Valvular Heart Disease. Eur Heart J 2003;24:1231-43.

22. Sawaya FJ, Deutsch MA, Seiffert M, et al. Safety and Efficacy of Transcatheter Aortic Valve Replacement in the Treatment of Pure Aortic Regurgitation in Native Valves and Failing Surgical Bioprostheses: Results From an International Registry Study. JACC Cardiovasc Interv 2017;10:1048-56.

23. Seiffert M, Diemert P, Koschyk D, et al. Transapical implantation of a second-generation transcatheter heart 
valve in patients with noncalcified aortic regurgitation. JACC Cardiovasc Interv 2013;6:590-7.

24. Ribeiro HB, Webb JG, Makkar RR, et al. Predictive factors, management, and clinical outcomes of coronary obstruction following transcatheter aortic valve implantation: insights from a large multicenter registry. J Am Coll Cardiol 2013;62:1552-62.

25. Ribeiro HB, Rodes-Cabau J, Blanke P, et al. Incidence, predictors, and clinical outcomes of coronary obstruction following transcatheter aortic valve replacement for degenerative bioprosthetic surgical valves: insights from the VIVID registry. Eur Heart J 2018;39:687-95.

26. Yamamoto M, Shimura T, Kano S, et al. Impact of preparatory coronary protection in patients at high anatomical risk of acute coronary obstruction during transcatheter aortic valve implantation. Int J Cardiol 2016;217:58-63.

27. Abramowitz Y, Chakravarty T, Jilaihawi H, et al. Clinical impact of coronary protection during transcatheter aortic valve implantation: first reported series of patients. EuroIntervention 2015;11:572-81.

28. Dvir D, Khan J, Kornowski R, et al. Novel strategies in aortic valve-in-valve therapy including bioprosthetic valve fracture and BASILICA. EuroIntervention 2018;14:AB74-82 .

29. Yudi MB, Sharma SK, Tang GHL, et al. Coronary Angiography and Percutaneous Coronary Intervention After Transcatheter Aortic Valve Replacement. J Am Coll Cardiol 2018;71:1360-78.

30. Aikawa Y, Kataoka Y, Kanaya T, et al. Procedural challenge of coronary catheterization for ST-segment elevation myocardial infarction in patient who underwent transcatheter aortic valve replacement using the CoreValve(TM). Cardiovasc Diagn Ther 2018;8:190-5.

31. Athappan G, Gajulapalli RD, Tuzcu ME, et al. A systematic review on the safety of second-generation transcatheter aortic valves. EuroIntervention 2016;11:1034-43.

32. Mathur M, Hira RS, Smith BM, et al. Fully Percutaneous Technique for Transaxillary Implantation of the Impella CP. JACC Cardiovasc Interv 2016;9:1196-8.

33. Greenbaum AB, Babaliaros VC, Chen MY, et al. Transcaval Access and Closure for Transcatheter Aortic Valve Replacement: A Prospective Investigation. J Am Coll Cardiol 2017;69:511-21.

34. Mylotte D, Osnabrugge RL, Martucci G, et al. Adoption Of Transcatheter Aortic Valve Implantation In Western Europe. Interv Cardiol 2014;9:37-40.
35. Cohen DJ. Cost-effectiveness of transcatheter vs. surgical aortic valve replacement in intermediate risk patients: results from the PARTNER 2A. TCT; Denver CO, 2018.

36. Kotronias RA, Teitelbaum M, Webb JG, et al. Early Versus Standard Discharge After Transcatheter Aortic Valve Replacement: A Systematic Review and Meta-Analysis. JACC Cardiovasc Interv 2018;11:1759-71.

37. Durko AP, Osnabrugge RL, Van Mieghem NM, et al. Annual number of candidates for transcatheter aortic valve implantation per country: current estimates and future projections. Eur Heart J 2018;39:2635-42.

38. Makkar RR, Fontana G, Jilaihawi H, et al. Possible Subclinical Leaflet Thrombosis in Bioprosthetic Aortic Valves. New Engl J Med 2015;373:2015-24.

39. Piccolo R, Pilgrim T, Franzone A, et al. Frequency, Timing, and Impact of Access-Site and Non-AccessSite Bleeding on Mortality Among Patients Undergoing Transcatheter Aortic Valve Replacement. JACC Cardiovasc Interv 2017;10:1436-46.

40. Rodes-Cabau J, Masson JB, Welsh RC, et al. Aspirin Versus Aspirin Plus Clopidogrel as Antithrombotic Treatment Following Transcatheter Aortic Valve Replacement With a Balloon-Expandable Valve: The ARTE (Aspirin Versus Aspirin + Clopidogrel Following Transcatheter Aortic Valve Implantation) Randomized Clinical Trial. JACC Cardiovasc Interv 2017;10:1357-65.

41. Maes F, Stabile E, Ussia GP, et al. Meta-Analysis Comparing Single Versus Dual Antiplatelet Therapy Following Transcatheter Aortic Valve Implantation. Am J Cardiol 2018;122:310-5.

42. Cerrato E, Nombela-Franco L, Nazif TM, et al. Evaluation of current practices in transcatheter aortic valve implantation: The WRITTEN (WoRldwIde TAVI ExperieNce) survey. Int J Cardiol 2017;228:640-7.

43. Kapadia SR, Kodali S, Makkar R, et al. Protection Against Cerebral Embolism During Transcatheter Aortic Valve Replacement. J Am Coll Cardiol 2017;69:367-77.

44. Giustino G, Sorrentino S, Mehran R, et al. Cerebral Embolic Protection During TAVR: A Clinical Event Meta-Analysis. J Am Coll Cardiol 2017;69:465-6.

Cite this article as: Neylon A, Ahmed K, Mercanti F, Sharif F, Mylotte D. Transcatheter aortic valve implantation: status update. J Thorac Dis 2018;10(Suppl 30):S3637-S3645. doi: $10.21037 /$ jtd.2018.10.34 